\title{
Intersection Theory and Higgs Physics
}

\author{
Hjalte Frellesvig* ${ }^{* \dagger}$ \\ Dipartimento di Fisica e Astronomia, Università di Padova, Via Marzolo 8, 35131 Padova, Italy \\ INFN, Sezione di Padova, Via Marzolo 8, 35131 Padova, Italy \\ E-mail: hjalte.frellesvigepd.infn.it
}

This is a contribution to the proceedings of the RADCOR2019 conference.

When finding linear relations between Feynman integrals using integration-by-parts identities, a very large linear system has to be solved as an intermediate step. This makes other approaches to the derivation of these identities a worthwhile pursuit. In this context, the concept of the intersection number is of interest, as it allows for the definition of (what amounts to) a scalar product between Feynman integrals, allowing the coefficients of the master integrals to be extracted using ordinary projections in the space of Feynman integrals. Using this novel method, we will derive and discuss a number of identities between Feynman integrals relevant for Higgs physics.

14th International Symposium on Radiative Corrections (RADCOR2019)

9-13 September 2019

Palais des Papes, Avignon, France

\footnotetext{
* Speaker.

${ }^{\dagger}$ These proceedings are based on work done with Federico Gasparotto, Stefano Laporta, Manoj K. Mandal, Pierpaolo Mastrolia, Luca Mattiazzi, and Sebastian Mizera.
}

(C) Copyright owned by the author(s) under the terms of the Creative Commons 


\section{Introduction}

Recently, new approach to the derivations of linear relations between Feynman integrals based on the mathematical concept of the intersection number has been developed, as an alternative to the traditional IBP-based method. While these proceedings are based on the publications [1,2], the application of Intersection theory to Feynman integrals was pioneered in ref. [3] by Mastrolia and Mizera. See also the proceedings by Manoj K. Mandal [4].
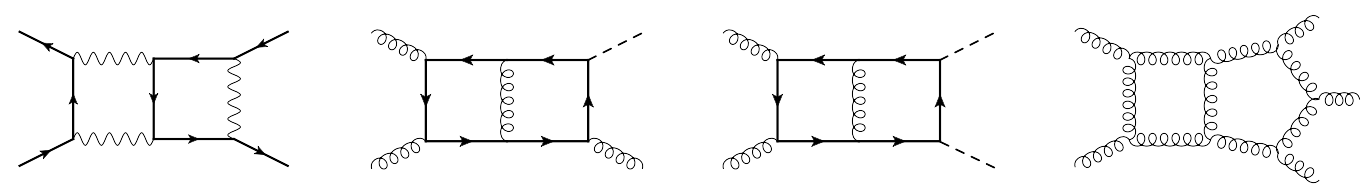

Figure 1: State-of-the-art scattering processes, each illustrated by a representative two-loop Feynman diagram. From left to right: Bhabha scattering, Higgs+jet production, double-Higgs production, and three-jet production.

The evaluation of Feynman integrals, is an essential part of state-of-the-art scattering amplitude computations, such as those illustrated in fig. 1. A Feynman integral is in general an object of the form

$$
I_{a_{1} \cdots a_{P} ; \cdots a_{n}}=\int \frac{\mathrm{d}^{d} k_{i}}{\pi^{d / 2}} \cdots \int \frac{\mathrm{d}^{d} k_{L}}{\pi^{d / 2}} \frac{N(k)}{D_{1}^{a_{1}}(k) D_{2}^{a_{2}}(k) \cdots D_{P}^{a_{P}}(k)}
$$

where the $D$ s are propagators of the form $D_{i}=(k+p)^{2}-m^{2}$, where $k$ and $p$ are $d$-dimensional momenta (internal and external). Additionally $N(k)$ is a numerator function, and the $a_{i}$ are integer powers.

Writing down all the Feynman diagrams, and performing the Dirac, Lorentz, and color algebra, will give an expression containing $\mathscr{O}(10000)$ such Feynman integrals for one of the state-of-theart processes. In principle they all have to be calculated in order to get a result for the scattering amplitude, but thankfully they are not all independent but are related by linear relation. The use off these relations can reduce the set of Feynman integrals that have to be computed to a minimal set of linearly independent integrals, known as master integrals, with a size of $\mathscr{O}(100)$ for one of the state-of-the-art processes - a much more manageable number.

The derivation of such linear relations are the main bottleneck in current amplitude calculations. The traditional approach to the derivation of such identities relies on the use of integrationby-parts identities [5] systematized by Laporta's algorithm [6] which is implemented in a number of public and private codes (in the following we will use FIRE [7] and Kira [8]). We will not here go through the details of that approach, merely mention that it relies on the IBP equation

$$
\int \frac{\mathrm{d}^{d} k}{\pi^{d / 2}} \frac{\partial}{\partial k^{\mu}} \frac{q^{\mu} N(k)}{D_{1}^{a_{1}}(k) \cdots D_{P}^{a_{P}}(k)}=0
$$

where varying $k, q$, and the $a_{i}$, yield different linear relations between Feynman integrals, and reducing the set of Feynman integrals that may appear in a given scattering amplitude calculation to master integrals, requires the solution of a huge linear system that may contain $\mathscr{O}\left(10^{7}\right)$ linear 
equation for a naive implementation. So even though a lot of effort has been put into optimizing the algorithm, solving a linear system of that size is never going to be fast, so to find a different way of deriving the linear relations seems highly desirable.

\section{Theory}

Writing a Feynman integral in terms of master integrals and their coefficients

$$
I=\sum_{i \in \text { masters }} c_{i} I_{i}
$$

seems reminiscent of writing a vector in a vector space in terms of basis-vectors of that space. In fact the correspondence is not merely metaphorical, the set of Feynman integrals forms a vector space in the mathematical sense. The existence of an inner product is not a requirement for forming a vector space, but if an inner product existed, the coefficients of the basis vectors could be extracted as [3]

$$
\begin{aligned}
\langle v| & =\sum_{i, j}\left\langle v v_{j}^{*}\right\rangle\left(C^{-1}\right)_{j i}\left\langle v_{i}\right| \quad \text { with } \quad C_{i j}=\left\langle v_{i} v_{j}^{*}\right\rangle \\
\Leftrightarrow c_{i} & =\sum_{j}\left\langle v v_{j}^{*}\right\rangle\left(C^{-1}\right)_{j i}
\end{aligned}
$$

For the proof of eq. (2.2) see e.g. refs. [1, 4], but notice that in the case of an orthonormal basis $C_{i j}=\delta_{i j}$, the formula above reduces to $c_{i}=\left\langle v v_{i}^{*}\right\rangle$ known from high-school.

This means that if there were some way to define (what amounts to) an inner product between Feynman integrals, it would be possible to extract the coefficients of the master integrals without going through the IBP equation and the corresponding huge linear systems.

To get to that point, we will need the Baikov representation for Feynman integrals. The Baikov representation [9] is a parametric representation ${ }^{1}$, which means that the integrations over the $d$ dimensional loop-momenta of eq. (1.1), get replaced with integrations over an integer number of scalar variables, here known as Baikov variables.

The Baikov representation is given as

$$
I=\int \frac{\mathrm{d}^{d} k_{1}}{\pi^{d / 2}} \cdots \int \frac{\mathrm{d}^{d} k_{L}}{\pi^{d / 2}} \frac{N(k)}{D_{1}^{a_{1}}(k) \cdots D_{P}^{a_{P}}(k)}=K \int_{\mathscr{C}} \mathrm{d}^{n} x \frac{\mathscr{B}^{\gamma}(x) N(x)}{x_{1}^{a_{1}} \cdots x_{P}^{a_{P}}}
$$

where $x_{i}$ are the Baikov variables, $\mathscr{B}$ is known as the Baikov polynomial, the integration contour is defined by the equation $\mathscr{C}=\{\mathscr{B}>0\}$, and the power $\gamma=(d-E-L-1) / 2$. Additionally $n=L(L+1) / 2+E L$ which is the same as the number of independent scalar product one can form between the $L$ loop-momenta and the $E$ independent external momenta. We will point out that the Baikov representation has the property that the propagators $D_{i}$ are in one-to-one correspondence with the Baikov variables $x_{i}$. The quadratic growth of the number of variables with the number of loops, makes the Baikov representation worse behaved that other parametric representations such

\footnotetext{
${ }^{1}$ Other examples of parametric representations are the Feynman representation, the Schwinger representation, and the Lee-Pomeransky representation.
} 
as Feynman parametrization. One way of remedying this quadratic growth a little is to use the loop-by-loop version [10] of Baikov parametrization

$$
I=\tilde{K} \int_{\mathscr{C}} \mathrm{d}^{\tilde{n}} x \frac{\left(\prod_{j=1}^{2 L-1} \mathscr{B}_{j}^{\gamma_{j}}(x)\right) N(x)}{x_{1}^{a_{1}} \cdots x_{P}^{a_{P}}}
$$

introduced by making a Baikov representation of each loop in a multi-loop Feynman integral individually, rather than parametrizing the whole integral at once. Doing this has the property that it might decrease the number of integration variables compared to the standard version of the parametrization $(\tilde{n} \leq n)$. This is a property we will use a lot in the following.

The reason for using the Baikov representation over one of the other parametric representations, is that it a natural representation for doing generalized unitarity cuts. A generalized unitarity cut is in this context to be understood as the contour deformation

$$
\int_{\mathscr{C}} \frac{f(x)}{x^{a}} \mathrm{~d} x \rightarrow \oint \frac{f(x)}{x^{a}} \mathrm{~d} x
$$

where the right hand side can be evaluated trivially using the residue theorem. We should highlight the fact that if $a \leq 0$ so the propagator is not present as a propagator, the value of the cut integral will be zero. The property of generalized unitarity cuts that will interest us here, is that even though they change the value of the integrals, they preserve the linear relations between them [11, 12], so relations such as eq. (2.1) are equally true before and after such a cut.

The Baikov representation can be re-written as

$$
I=\int_{\mathscr{C}} \mathrm{d}^{n} x \frac{\mathscr{B}^{\gamma}(x) N(x)}{x_{1}^{a_{1}} \cdots x_{P}^{a_{P}}}=\int_{\mathscr{C}} u \phi
$$

where

$$
u=\mathscr{B}^{\gamma} \quad \text { and } \quad \phi=\frac{N(x)}{x_{1}^{a_{1}} \cdots x_{P}^{a_{P}}} \mathrm{~d} x_{1} \wedge \cdots \wedge \mathrm{d} x_{n}
$$

are defined such that $u$ is a multi-valued function (since $\gamma$ non-integer in dimensional regularization) and $\phi$ is a rational function times a differential $n$-form, and this definition carries over to the loopby-loop representation as well. Rewriting $I$ further, we can can express is as [3]

$$
\left.I=\int_{\mathscr{C}} u \phi={ }_{\omega}\langle\phi| \mathscr{C}\right]
$$

where $\omega=\mathrm{d} \log (u)$ is known as the twist. In this notation $\mathscr{C}$ and $\phi$ should be understood as representatives of a twisted cycle and a twisted co-cycle respectively, which are defined as equivalence classes of those integration contours (for $\mathscr{C}$ ) and differential forms (for $\phi$ ) that integrate to the same result, given a specific $u$ [3]. The set of twisted co-cycles is known as the twisted cohomology group, and we realize that the number of master integral will be the dimension of the twisted cohomology group, as it is nothing but the number of different integrands that integrate to genuinely different results. From this fact one may derive the Lee-Pomeransky criterion [13] for the number of master integrals $v$, which says that

$$
v=\text { the number of solutions to " } \omega=0 "
$$


something we will use in the following.

This bring us to the intersection number $\langle\phi \mid \xi\rangle$. This is also defined as a pairing, but rather than between a twisted cycle and co-cycle, it is between a twisted co-cycle $\phi$ and a dual twisted cocycle $\xi$, and this object has exactly the properties of a scalar product that we have been looking for. For the mathematical definitions of $\xi$, its associated dual cohomology group, and the intersection number itself, see e.g. refs. [14, 15, 16], but let us here merely write an expression for it valid in the univariate case:

$$
\langle\phi \mid \xi\rangle_{\omega}=\sum_{p \in \mathscr{P}} \operatorname{Res}_{z=p}(\psi \xi) \quad(\mathrm{d}+\omega) \psi=\phi
$$

where $\mathscr{P}$ is defined as the set of poles of $\omega$. One might fear that to compute $\psi$ would involve the genuine solution of a differential equation, but since the result has to be used only inside the residue, it is sufficient to use a series ansatz around the point where the residue is taken

$$
\psi \rightarrow \psi_{p}=\sum_{i=\min }^{\max } \kappa_{i}(z-p)^{i}
$$

making sure that 'min' and 'max' are chosen such that all the terms that may contribute to the residue are included.

With these definitions in mind, we may now write down the expression for the coefficients in the master integral decomposition. It is as given [3] by eq. (2.3) as

$$
\begin{aligned}
\langle\phi| \mathscr{C}] & \left.=\sum_{i=1}^{v} c_{i}\left\langle\phi_{i}\right| \mathscr{C}\right] \Rightarrow \\
c_{i} & =\sum_{j=1}^{v}\left\langle\phi \mid \xi_{j}\right\rangle\left(C^{-1}\right)_{j i} \quad \text { with } \quad C_{i j}=\left\langle\phi_{i} \mid \xi_{j}\right\rangle
\end{aligned}
$$

where the scalar product is to be understood as the intersection number which in the univariate case is given by eq. (2.11).

\section{Example: the double box}

Let us as an example show the decomposition of the massless double box (see fig. 2) on the maximal $^{2}$ cut, following ref. [1].

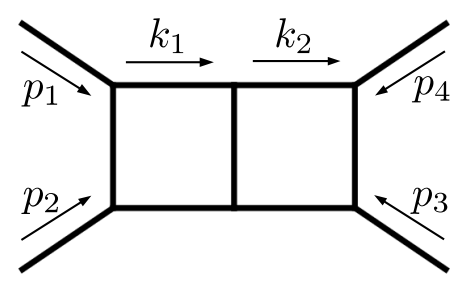

Figure 2: The massless double box

\footnotetext{
${ }^{2}$ Different people mean different things with the phrase "maximal cut". In these proceedings it is going to mean cutting all the propagators that are actually there, so 7 for the case of the double box.
} 
The double-box is defined by the seven propagators

$$
\begin{array}{lll}
D_{1}=k_{1}^{2}, & D_{2}=\left(k_{1}-p_{1}\right)^{2}, & D_{3}=\left(k_{1}-p_{1}-p_{2}\right)^{2}, \quad D_{4}=\left(k_{1}-k_{2}\right)^{2}, \\
D_{5}=\left(k_{2}-p_{1}-p_{2}\right)^{2}, & D_{6}=\left(k_{2}+p_{4}\right)^{2}, & D_{7}=k_{2}^{2} .
\end{array}
$$

and additionally the kinematics is such that $p_{i}^{2}=0,\left(p_{1}+p_{2}\right)^{2}=s$, and $\left(p_{1}+p_{4}\right)^{2}=t$. In the loopby-loop Baikov representation eight variables are needed, so the above set has to be supplemented by one additional Baikov variable

$$
z=\left(k_{2}-p_{1}\right)^{2}
$$

allowing us to write the double-box as an eight-fold integral. But on the maximal cut, seven of those integrations become trivial, leaving us with a one-fold integral

$$
I_{7 \times \mathrm{cut}}=\int_{\mathscr{C}} u_{7 \times \mathrm{cut}} \phi \quad \text { with } \quad u_{7 \times \mathrm{cut}}=z^{d / 2-3}(z+s)^{2-d / 2}(z-t)^{d-5}
$$

From there we can compute

$$
\omega=\mathrm{d} \log (u)=\left(\frac{d-6}{2 z}+\frac{4-d}{2(z+s)}+\frac{d-5}{z-t}\right) \mathrm{d} z
$$

and we see that $\omega=0$ has two solutions, corresponding to there being $v=2$ master integrals surviving on the maximal cut, a result known to be correct from the literature [17].

Let us try to perform a reduction, using eq. (2.13), of the integral with two powers of the ISP $z$ in the numerator, to a basis of master integrals with zero and one powers respectively. This means we want to find the coefficients $c_{1}$ and $c_{2}$ in the relation

$$
I_{111111 ;-2}=c_{1} I_{1111111 ; 0}+c_{2} I_{1111111 ;-1}+\text { lower }
$$

To use eq. (2.13) we need expressions for the co-cycles and dual co-cycles. The co-cycles $\phi$ are fixed by the problem, and are given by

$$
\phi=z^{2} \mathrm{~d} z, \quad \phi_{1}=1 \mathrm{~d} z, \quad \phi_{2}=z \mathrm{~d} z .
$$

For the dual co-cycles $\xi$ we are free to pick almost whatever we like, but it turns out that picking a $\mathrm{d} \log$-form for the co-cycles simplifies intermediate expressions. We pick

$$
\xi_{1}=\left(\frac{1}{z}-\frac{1}{z+s}\right) \mathrm{d} z, \quad \xi_{2}=\left(\frac{1}{z+s}-\frac{1}{z-t}\right) \mathrm{d} z
$$

We may then compute the intersection numbers between the co-cycles and dual co-cycles using eq. (2.11). We will not go into further details with the computation, merely state the results

$$
\begin{array}{rlrl}
\left\langle\phi \mid \xi_{1}\right\rangle & =\frac{s\left(4(d-5) t^{2}-3(d-4)(3 d-14) s^{2}-4(d-5)(2 d-9) s t\right)}{4(d-5)(d-4)(d-3)}, & \\
\left\langle\phi \mid \xi_{2}\right\rangle & =\frac{s(s+t)(3(d-4)(3 d-14) s+2(d-6)(d-5) t)}{4(d-5)(d-4)(d-3)}, & & \\
\left\langle\phi_{1} \mid \xi_{1}\right\rangle & =\frac{-s}{d-5}, & \left\langle\phi_{1} \mid \xi_{2}\right\rangle & =\frac{s+t}{d-5}, \\
\left\langle\phi_{2} \mid \xi_{1}\right\rangle & =\frac{s((3 d-14) s+2(d-5) t)}{2(d-5)(d-4)}, & \left\langle\phi_{2} \mid \xi_{2}\right\rangle & =\frac{-(3 d-14) s(s+t)}{2(d-5)(d-4)} .
\end{array}
$$

Combining these according to eq. (2.13) gives the results

$$
c_{1}=\frac{(d-4) s t}{2(d-3)}, \quad c_{2}=\frac{2 t-3(d-4) s}{2(d-3)},
$$

in agreement with public codes such as FIRE [7]. This shows that our approach to integral decomposition works. 


\section{Further examples}
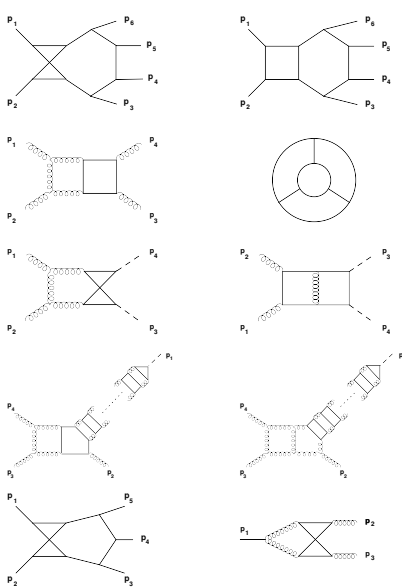
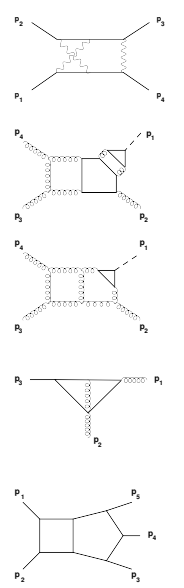
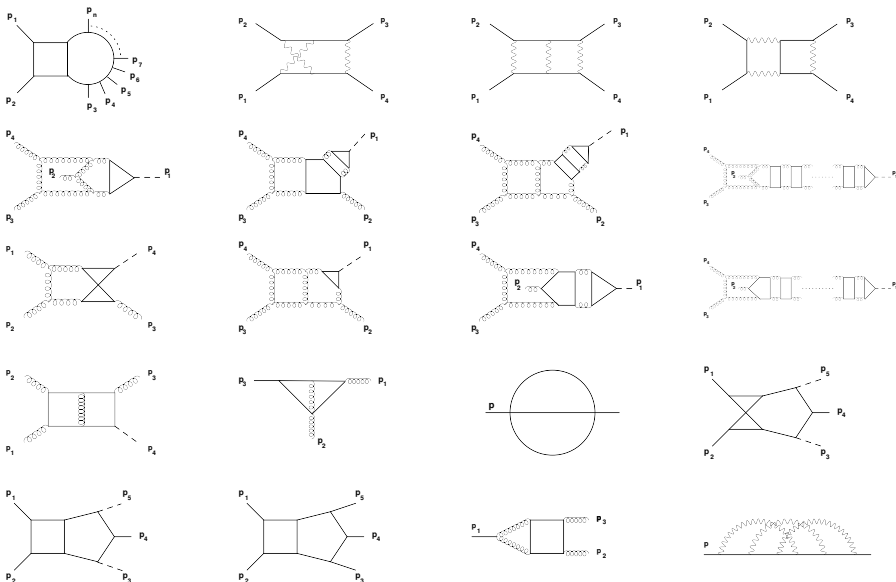
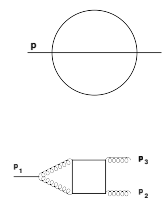
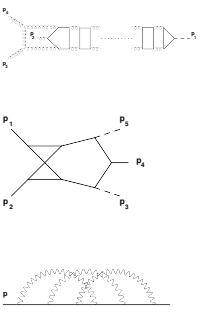

Figure 3: Thirty examples of integrals for which we did the reduction on the maximal cut, in ref. [1]. Please note that while there are examples of integrals with an arbitrary number of loops or legs respectively, one thing that all the integrals in this figure have in common, is that the loopby-loop Baikov representation allows for a univariate expression after the maximal cut has been performed.

In ref. [1] we did a lot of additional examples of reductions on the maximal cut (see fig. 3). It may be seen that many of the integrals are of a type relevant for Higgs production, giving the reason for title of these proceedings, and the talk on which they are based. Let us focus on one example - that of the third integral in the fourth row of fig. 3, relevant for NLO QCD $H+j$ production retaining the full dependence on the masses of the Higgs and the internal quark (see ref. [18]). As before, the loop-by-loop Baikov representations requires the introduction of one extra variable, and on the maximal cut that integral may be written as eq. (3.3) but with

$$
\begin{aligned}
u_{7 \times \mathrm{cut}}= & z^{d-5}\left(z^{2}+s z+m_{t}^{2} s\right)^{\frac{4-d}{2}} \\
& \times\left(\left(m_{H}^{2}-s\right)^{2} z^{2}+2\left(m_{H}^{2}-s\right) s t z+s t\left(4 m_{t}^{2}\left(m_{H}^{2}-s-t\right)+s t\right)\right)^{\frac{d-5}{2}}
\end{aligned}
$$

Here eq. (2.10) gives $v=4$, again corresponding to the result in the literature. One example of a reduction unto master integrals is

$$
I_{1111111 ;-1}=c_{1} I_{1111111 ; 0}+c_{2} I_{1211111 ; 0}+c_{3} I_{1111211 ; 0}+c_{4} I_{1111112 ; 0}+\text { lower }
$$

and again the expressions for the $c_{i}$ may be computed using eq. (2.13) giving results in agreement with the public codes.

\section{The multivariate case}

Aside from eq. (2.11), there is nothing in the previous that is restricted to the univariate case or to maximal cuts. In particular the projection formula eq. (2.13) has exactly the same form in the multivariate case, the only difference is that the $\langle\phi \mid \xi\rangle$ have to be interpreted as multivariate 
intersection numbers. Multivariate intersection numbers are discussed in refs. [15, 19, 2] and based on the formulation in ref. [2], an $n$-variate intersection number $\mathbf{n}\langle\phi \mid \xi\rangle$ is specified by the following equations (5.1) to (5.5)

$$
\begin{gathered}
{ }_{\mathbf{n}}\left\langle\phi^{(\mathbf{n})} \mid \xi^{(\mathbf{n})}\right\rangle=-\sum_{p \in \mathscr{P}_{n}} \operatorname{Res}_{n}\left(\mathbf{n}-\mathbf{1}\left\langle\phi^{(\mathbf{n})} \mid h_{i}^{(\mathbf{n}-\mathbf{1})}\right\rangle \psi_{i}^{(n)}\right), \\
\left(\delta_{i j} \partial_{z_{n}}-\hat{\Omega}_{i j}^{(n)}\right) \psi_{j}^{(n)}=\hat{\xi}_{i}^{(n)}, \\
\hat{\Omega}_{i j}^{(n)}=-\left(\mathbf{C}_{(\mathbf{n}-\mathbf{1})}^{-1}\right)_{i k} \mathbf{n}-\mathbf{1}\left\langle e_{k}^{(\mathbf{n}-\mathbf{1})} \mid\left(\partial_{z_{n}}-\hat{\omega}_{n}\right) h_{j}^{(\mathbf{n}-\mathbf{1})}\right\rangle, \\
\xi_{i}^{(n)}=\left(\mathbf{C}_{(\mathbf{n}-\mathbf{1})}^{-1}\right)_{i j} \mathbf{n}-\mathbf{1}\left\langle e_{j}^{(\mathbf{n}-\mathbf{1})} \mid \xi^{(\mathbf{n})}\right\rangle \\
\left(\mathbf{C}_{(\mathbf{n}-\mathbf{1})}\right)_{i j} \equiv_{\mathbf{n}-\mathbf{1}}\left\langle e_{i}^{(\mathbf{n}-\mathbf{1})} \mid h_{j}^{(\mathbf{n}-\mathbf{1})}\right\rangle .
\end{gathered}
$$

We will not go through in detail how these equations should be understood or applied, merely point out a few features: We see that the expression for the intersection number, eq. (5.1) is given as a sum over residues exactly as in the univariate case of eq. (2.11). Additionally we see that inside the residue appears an object $\psi$ defined as the solution to a differential equation eq. (5.2) which in principle can be solved with a series ansatz as in the univariate case. Finally we notice that the expression in recursive, in the sense that the $n$-variate intersection number of eq. (5.1) in given in terms of $(n-1)$-variate intersection numbers, and since we know how to evaluate a univariate intersection number, the recursion will eventually end.
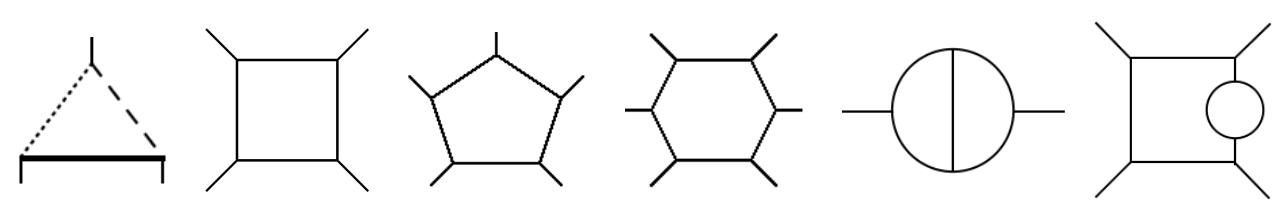

Figure 4: Those Feynman integrals for which we in ref. [2] have done the complete reduction using the intersection-based approach.

If ref. [2] we did the complete reduction of a number of one- and two-loop Feynman integrals, using the intersection-based approach (see fig. 4). Let us show as an example the simplest of them all, the massless one-lop box. We want to do the reduction

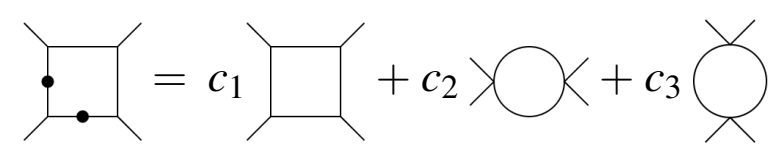

where the dot denotes a squared propagator. The integrals in this integral family, without any unitarity cuts, may be expressed as $\int u \phi$ with

$$
\begin{aligned}
u(\mathbf{x})= & \left(\left(s t-s x_{4}-t x_{3}\right)^{2}-2 t x_{1}\left(s\left(t+2 x_{3}-x_{2}-x_{4}\right)+t x_{3}\right)\right. \\
& \left.+s^{2} x_{2}^{2}+t^{2} x_{1}^{2}-2 s x_{2}\left(t\left(s-x_{3}\right)+x_{4}(s+2 t)\right)\right)^{\frac{d-5}{2}} .
\end{aligned}
$$


and

$$
\hat{\phi}=\left(x_{1}^{2} x_{2}^{2} x_{3} x_{4}\right)^{-1}, \hat{\phi}_{1}=\left(x_{1} x_{2} x_{3} x_{4}\right)^{-1}, \hat{\phi}_{2}=\left(x_{1} x_{3}\right)^{-1}, \hat{\phi}_{3}=\left(x_{2} x_{4}\right)^{-1},
$$

with $\phi=\hat{\phi} \bigwedge_{i} \mathrm{~d} x_{i}$. Introducing regulators ${ }^{3} u \rightarrow u \prod_{i} x_{i}^{\rho_{i}}$ where the $\rho_{i}$ are to be put to zero at the end of the computation, we may compute $\omega$, and from there we obtain $v=3$ as it is seen in eq. (5.6) and known to be the correct number. Applying the projection formula eq. (2.13) with the multivariate intersection numbers as they may be computed from eqs. (5.1) to (5.5), we get the coefficients

$$
c_{1}=\frac{(d-5)(d-6)}{s t}, \quad c_{2}=\frac{-4(d-5)(d-3)}{s^{3} t}, \quad c_{3}=\frac{-4(d-5)(d-3)}{s t^{3}},
$$

in agreement with the public codes.

\section{Discussion}

In ref. [2] we propose a different algorithm for multivariate reductions that the one presented above. This algorithm we call the bottom-up approach and it works by doing the reductions not on the full multivariate expression, but on a set of "spanning cuts" [20] chosen such that each master integral is non-vanishing on at least one of the cuts. In the one-loop box example above, this would be the cuts corresponding to the two bubbles. Such an algorithm would involve more uses of the projection formula eq. (2.13), but this is counteracted by the fact that the intersection numbers involved are of fewer variables, making their computation simpler, something that will most likely be a worthwhile trade-off in more complicated examples. But in spite of this, it is still an open question what algorithm is the most optimal for the derivation of a complete reduction. Also a worthwhile investigation is into ways of calculating multivariate intersection numbers other than eqs. (5.1) to (5.5), that perhaps will be easier or more computationally effective.

The utility of the intersection based approach is not limited to the kind of linear relations given by eq. (2.1). In refs. $[3,1,2]$ it is also discussed how to use the technology to express the right hand sides of differential equations (in kinematic variables) and of dimension shift relations. Focusing on the former case we have

$$
\partial_{s} I=\int_{\mathscr{C}} \partial_{s}(u \phi)=\int_{\mathscr{C}} u \tilde{\phi} \quad \text { with } \quad \tilde{\phi}=\partial_{s} \phi+\phi\left(\partial_{s} u\right) / u
$$

where $\tilde{\phi}$ is a form with rational pre-factor, that may be reduced using eq. (2.13) as the other cases we have seen. Likewise the approach does also work for purely mathematical functions such as the hypergeometric function ${ }_{2} F_{1}$ and its generalisations, where it can be used to deduce linear relations (known as contiguity relations in that context) between functions with different values of their arguments.

Directions worth exploring in the future may be connection to the co-product for Feynman integrals and special functions (see e.g. refs. [21,22]) and to the $\varepsilon$-expansions of Feynman integrals (see ref. [23]), but at least from the speakers perspective the most promising potential development would be that of a completely general computer implementation of the reduction algorithm

\footnotetext{
${ }^{3}$ This has to be done to ensure that all poles of $\phi$ are poles of $\omega$, which is a requirement for the intersection theory, in the way we use it, to be valid.
} 
described in the above, since there is much reason to believe that such an implementation would be much more efficient that those based on IBPs, as the huge linear systems that have to be solved as an intermediate step in the IBP based approach, are avoided completely.

\section{Acknowledgments}

The work of H.F. is part of the HiProLoop project funded by the European Union's Horizon 2020 research and innovation programme under the Marie Skłodowska-Curie grant agreement 74717 .

\section{References}

[1] H. Frellesvig, F. Gasparotto, S. Laporta, M. K. Mandal, P. Mastrolia, L. Mattiazzi, and S. Mizera, Decomposition of Feynman Integrals on the Maximal Cut by Intersection Numbers, JHEP 05 (2019) 153, [arXiv:1901.11510].

[2] H. Frellesvig, F. Gasparotto, M. K. Mandal, P. Mastrolia, L. Mattiazzi, and S. Mizera, Vector Space of Feynman Integrals and Multivariate Intersection Numbers, Phys. Rev. Lett. 123 (2019), no. 20 201602, [arXiv:1907.02000].

[3] P. Mastrolia and S. Mizera, Feynman Integrals and Intersection Theory, JHEP 02 (2019) 139, [arXiv:1810.03818].

[4] M. K. Mandal, Decomposition of Feynman Integrals on the Maximal Cut by Intersection Numbers, .

[5] K. G. Chetyrkin and F. V. Tkachov, Integration by Parts: The Algorithm to Calculate beta Functions in 4 Loops, Nucl. Phys. B192 (1981) 159-204.

[6] S. Laporta, High precision calculation of multiloop Feynman integrals by difference equations, Int. J. Mod. Phys. A15 (2000) 5087-5159, [hep-ph/ 0102033$].$

[7] A. V. Smirnov, FIRE5: a C++ implementation of Feynman Integral REduction, Comput. Phys. Commun. 189 (2015) 182-191, [arXiv: 1408 .2372].

[8] P. Maierhöfer, J. Usovitsch, and P. Uwer, Kira - A Feynman integral reduction program, Comput. Phys. Commun. 230 (2018) 99-112, [arXiv: 1705.05610].

[9] P. A. Baikov, Explicit solutions of the multiloop integral recurrence relations and its application, Nucl. Instrum. Meth. A389 (1997) 347-349, [hep-ph/ 9611449 ].

[10] H. Frellesvig and C. G. Papadopoulos, Cuts of Feynman Integrals in Baikov representation, JHEP 04 (2017) 083, [arXiv:1701.07356].

[11] A. Primo and L. Tancredi, On the maximal cut of Feynman integrals and the solution of their differential equations, Nucl. Phys. B916 (2017) 94-116, [arXiv: 1610 . 08397].

[12] J. Bosma, M. Sogaard, and Y. Zhang, Maximal Cuts in Arbitrary Dimension, JHEP 08 (2017) 051, [arXiv:1704.04255].

[13] R. N. Lee and A. A. Pomeransky, Critical points and number of master integrals, JHEP 11 (2013) 165, [arXiv:1308.6676].

[14] K. Cho and K. Matsumoto, Intersection theory for twisted cohomologies and twisted Riemann's period relations I, Nagoya Math. J. 139 (1995) 67-86. 
[15] K. Matsumoto, Intersection numbers for logarithmic k-forms, Osaka J. Math. 35 (1998), no. 4 873-893.

[16] S. Mizera, Scattering Amplitudes from Intersection Theory, Phys. Rev. Lett. 120 (2018), no. 14 141602, [arXiv:1711.00469].

[17] V. A. Smirnov, Analytical result for dimensionally regularized massless on shell double box, Phys. Lett. B460 (1999) 397-404, [hep-ph/ 9905323 ].

[18] R. Bonciani, V. Del Duca, H. Frellesvig, J. M. Henn, F. Moriello, and V. A. Smirnov, Two-loop planar master integrals for Higgs $\rightarrow 3$ partons with full heavy-quark mass dependence, JHEP 12 (2016) 096, [arXiv:1609.06685].

[19] S. Mizera, Aspects of Scattering Amplitudes and Moduli Space Localization. PhD thesis, Perimeter Inst. Theor. Phys., 2019. arXiv:1906.02099.

[20] K. J. Larsen and Y. Zhang, Integration-by-parts reductions from unitarity cuts and algebraic geometry, Phys. Rev. D93 (2016), no. 4 041701, [arXiv: 1511.01071$].$

[21] S. Abreu, R. Britto, C. Duhr, E. Gardi, and J. Matthew, From positive geometries to a coaction on hypergeometric functions, arXiv:1910.08358.

[22] R. Britto, Generalized hypergeometric functions and intersection theory for Feynman integrals, .

[23] S. Mizera and A. Pokraka, From Infinity to Four Dimensions: Higher Residue Pairings and Feynman Integrals, arXiv:1910.11852. 